\title{
Features of aura in paediatric migraine diagnosed using the ICHD 3 beta criteria
}

Cephalalgia

$0(0)$ I-6

(C) International Headache Society 2017 Reprints and permissions: sagepub.co.uk/journalsPermissions.nav DOI: |0.1 I77/03331024|774857| journals.sagepub.com/home/cep

(S)AGE

\author{
Martina Balestri ${ }^{1} *$, Laura Papetti ${ }^{1} *$, Daniela Maiorani ${ }^{2}$, \\ Alessandro Capuano', Samuela Tarantino', Barbara Battan', \\ Federico Vigevano' and Massimiliano Valeriani ${ }^{1,3}$
}

\begin{abstract}
Background: In children and adolescents, the prevalence rate of migraine with aura is $1.6 \%$. Few studies concerning migraine with aura features in paediatric population have been reported.

Aim: The aim of our study was to investigate clinical features of aura in a retrospective cohort of children with migraine with aura. Furthermore, we studied whether the International Classification of Headache Disorder (ICHD) 3 beta version criteria could efficiently detect migraine with aura in a paediatric population.

Results: We included 164 patients who experienced aura associated with headache (mean age $9.92 \pm 2.64$ years). When the ICHD-II criteria were used, a final diagnosis of migraine with typical aura was obtained in I5.3\% of patients, probable migraine with typical aura in $13.4 \%$, and typical aura with headache in $61.8 \%$, while in in $9.5 \%$ of patients the diagnosis was undetermined. According to ICHD-3 beta, we diagnosed migraine with typical aura in $77.7 \%$ of patients, probable migraine with typical aura in $13.4 \%$, and an undetermined diagnosis in $9.5 \%$ (less than two attacks).

Conclusion: Aura features did not depend on age and were similar to those of adults. However, the headache could be difficult to classify if headache duration was considered. In this view, the ICHD-3 beta offers the advantage of not considering headache features, including pain duration, for the diagnosis of migraine with typical aura, thus making this diagnosis easier in children and adolescents.
\end{abstract}

\section{Keywords}

Migraine, aura, childhood, headache

Date received: 22 July 20I7; revised: 12 September 20I7; 27 September 20I7; accepted: 2 October 2017

\section{Introduction}

Migraine with aura (MWA) is described in the International Classification of Headache Disorders (ICHD) 3 edition (beta version) as "recurrent attacks, lasting minutes, of unilateral fully reversible visual, sensory or other central nervous system symptoms that usually develop gradually and are usually followed by headache and associated with migraine symptoms. Aura symptoms spreads gradually over 5 to $60 \mathrm{~min}$ utes... and are accompanied, or followed within 60 minutes, by headache" (1). Though less commonly, headache may be completely absent.

The prevalence of migraine in children and adolescents has been reported to be between 3 and 23\% (2-6). MWA prevalence is substantially lower, with an average rate of $1.6 \%$ (7). Patients with migraine attacks with aura could also have attacks without aura (1). Few studies concerning MWA features in a paediatric population are reported $(8,9)$.
Compared to the ICHD-I classification, the ICHD-II criteria have increased the sensitivity of the diagnosis of migraine without aura (MOA) in children from $21 \%$ to $53 \%(10,11)$. However, these criteria still failed to detect about half of paediatric migraine, particularly due to the difficulty in distinguishing tension-type headache from migraine in young children (7). In the third edition of the

\footnotetext{
'Headache Centre, Bambino Gesù Children Hospital, IRCCS, Rome, Italy ${ }^{2}$ Department of Pediatrics, Belcolle Hospital, Viterbo, Italy

${ }^{3}$ Centre for sensory motor interaction, Aalborg University, Aalborg, Denmark

*These authors contributed equally to this work.
}

\section{Corresponding author:}

Massimiliano Valeriani, Ospedale Pediatrico Bambino Gesù, IRCCS, Piazza Sant'Onofrio 4, 00165 Rome, Italy.

Email: massimiliano.valeriani@opbg.net 
ICHD criteria, the duration of the migraine attack in children and adolescent was shifted from 1-72 hours to 2-72 hours (1), being inadequate especially for young children (12).

The latest version of the ICHD criteria proposes a major novelty for the diagnosis of MWA. While the ICHD-II distinguished MTA (migraine with typical aura) from typical aura with headache (THA) depending on pain features (migrainous or not), in the ICHD-3 beta the headache features are not considered for the diagnosis of MTA. In particular, in the section concerning MTA (1), the ICHD-3 beta recognises two entities: Typical aura with headache and typical aura without headache (1). So, when the aura criteria are met, MTA can be diagnosed independently of whether the pain meets the criteria of migraine. This could be of great benefit for the diagnosis of MWA in paediatric patients, as it allows us to overtake the limitations of the previous criteria concerning the duration of pain (12).

The aims of our study were: a) To investigate the clinical characteristics of aura in a retrospective cohort of migraine children experiencing aura symptoms, and b) to compare ICHD-II and the ICHD-3 beta criteria for the diagnosis of MWA in a paediatric population.

\section{Materials and methods}

\section{Selection and classification of the patients}

We retrospectively reviewed the charts of patients attending the Headache Centre of Bambino Gesù Pediatric Hospital in a period from January 2002 to December 2011.

Patients who presented with neurological symptoms suggesting aura were selected, and those in whom a primary headache was diagnosed were included. In particular, the diagnosis of primary headache was based on the following main elements: a) all selected children were followed for at least 1 year and no different headache aetiology could be identified in this period, b) all children had a normal neurological examination, including fundus oculi examination, through the entire period considered, c) all children younger than 5 years had a neuroradiological examination (brain MRI or CT scan), which had to be normal. Other elements for the diagnosis of primary headache were: a) at least one relative with primary headache (first-degree relatives), and b) the coexistence or not of the so-called childhood periodic syndromes and related symptoms (cyclical vomiting syndrome, abdominal migraine, benign paroxysmal vertigo, benign paroxysmal torticollis, limb pain and motion sickness) $(13,14)$. None of the included children suffered from diseases potentially associated with secondary headache.

We considered pain localisation (orbital, frontal, temporal, vertex, parietal, occipital or diffuse), quality of pain (throbbing, constrictive, trafictive or dull), seven classes of duration of pain ( $<5$ minute; 5-30 minutes; 30 minutes to 1 hour; $1-2$ hours; $2-4$ hours; 4-24 hours and $>24$ hours), intensity (from 1 to 3 ), frequency of attacks (sporadic, multi-monthly, monthly, multi-weekly and weekly), and vegetative symptoms associated such as nausea, vomiting, photo and phonophobia (none of these, only 1, 2, 3 or 4). Aura characteristics included: Type (visual, sensitivity, motor or aphasia), duration (less than 5 minutes, between 5 and 10 minutes, between 10 and 30 minutes, between 30 minutes and 1 hour and more than 2 hours) and timing of aura manifestations with respect to headache (pre-ictal: 5-60 minutes before headache, ictal: Less than 5 minutes before headache; or post-ictal: Within 60 minutes after headache).

The final diagnosis of headache was made according to either the ICHD-II or ICHD-3 beta criteria and the results were compared. Diagnosis of migraine with typical aura (MTA) was made when the ICHD criteria were fully satisfied, while probable migraine with typical aura (pMTA) was diagnosed when the ICHD criteria were partially satisfied. When we used the ICHD-II criteria, typical aura with headache (TAH) was diagnosed when aura criteria were met, but headache pain did not fulfil the criteria for migraine. The patients in which neither the criteria for aura nor those for migraine were satisfied were classified as undetermined.

\section{Statistical analysis}

Statistical analysis was conducted by using the dedicated software MYSTAT 12 (v.12.02.). We used a $\chi^{2}$ test for the correlation analysis and a logistic regression for the regression analysis. Values of $p<0.05$ were considered significant.

Characteristics of aura (type, duration, and timing of onset with respect to headache) were compared among different groups of ages (less than 6 years, between 7-10 years, between 11 and 14 years, more than 15 years) using the $\chi^{2}$ test.

Regression analysis was used to verify significant correlation between diagnoses (MTA, pMTA, THA, undetermined) and headache features (duration of headache, pain localisation, quality of pain, intensity and vegetative accompanying symptoms); between diagnosis and aura features (type, timing, and duration of aura); between aura characteristics and headache features, and between age/gender and aura features. 


\section{Results}

We included 164 patients who experienced aura associated to headache (37.8\% males and $62.2 \%$ females). The mean age of headache onset was $9.92 \pm 2.64$ years (range: 5-17 years).

Pain localisation was frontal $(43.7 \%)$, temporal $(35.3 \%)$, orbital $(10 \%)$, diffuse $(3.3 \%)$, parietal $(2.5 \%)$, referred to vertex $(4.2 \%)$ and occipital $(0.8 \%)$. Headache quality was reported as: Throbbing in $52.6 \%$, constrictive in $37.8 \%$, trafictive in $6.4 \%$ and dull in $3.2 \%$ of cases. Attack duration was between 1 and 2 hours in $34.1 \%$ of the patients, between 5 and 30 minutes in $20.1 \%$, less than 5 minutes in $12.8 \%$, between 2 and 4 hours in $14.6 \%$, between 30 minutes and 1 hour in $12.8 \%$, more than 24 hours in $4.3 \%$ and between 4 and 24 hours in $1.2 \%$. Despite the main duration of headache being between 1 and 2 hours both in males and in females, pain duration was shorter than 1 hour in $55.4 \%$ of males and $36.1 \%$ of females $(p<0.005)$, while it was longer than 2 hours in only $14.9 \%$ of males and $25.8 \%$ of females $(p>0.005)$. Intensity of pain was in a range between a minimum of 1 and a maximum of 3 ; the mean value was $2.66 \pm 0.572$. Attack frequencies were grouped in five categories: Sporadic $(44.5 \%)$, monthly $(28 \%)$, multiweekly $(13.4 \%)$, weekly $(9.8 \%)$ and multi-monthly $(4.3 \%)$. We also considered the associated vegetative symptoms that may accompany headache (nausea, vomiting, photophobia and phonophobia). Two symptoms were reported by $30.7 \%$ of patients, three symptoms in $23.5 \%$, one symptom in $15 \%$ and all of them in $11.8 \%$. None of these conditions were referred to by $18.9 \%$ of patients. Photophobia was the most prevalent associated symptom $(64.6 \%)$, followed by phonophobia $(55.5 \%)$, nausea $(36 \%)$ and vomiting $(30.5 \%)$. Headache features are summarised in Figure 1.

Visual symptoms were reported in most attacks with aura $(93.2 \%)$ and included positive (bright spots, flashes of light or scintillating scotoma) and negative (loss of vision) phenomena. Somatosensory $(5.5 \%)$, motor $(0.6 \%)$, and aphasic $(0.6 \%)$ auras were less represented in our patients. Forty-two patients $(25.1 \%)$ experienced a second type of aura with somatosensory (64.3\%), speech $(23.8 \%$ : $14.3 \%$ dysarthria and 9.5\% aphasia), and motor (11.9\%) disturbances. Aura duration was less than 5 minutes in $18.8 \%$ of patients, between 5 and 10 minutes in $41.3 \%$, between 10 and 30 minutes in $28.3 \%$, between 30 minutes and $60 \mathrm{~min}-$ utes in $10.1 \%$, and more than 120 minutes in $1.4 \%$. Pre-ictal aura was present in $69.1 \%$ of patients, ictal aura in $21.1 \%$ and post-ictal aura in $9.7 \%$. Characteristics of aura in the different age groups are reported in Tables 1-3. Features of aura did not show significant differences when different ages have been considered $(p>0.05)$.

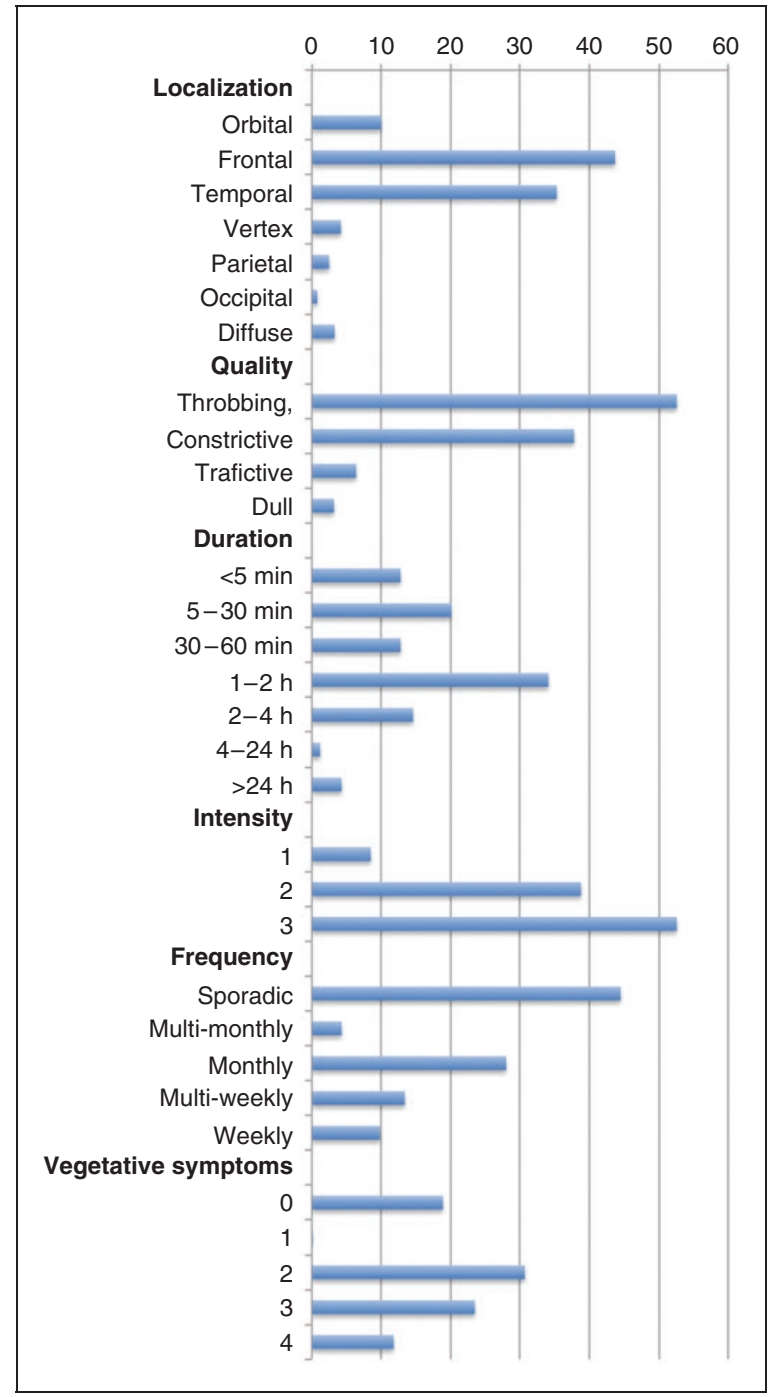

Figure I. Headache characteristics (\% of patients). Intensity: I mild; 2 moderate; 3 severe; vegetative symptoms (nausea, vomiting, photophobia and phonophobia): 0: None; I: One symptom; 2: Two associated symptoms; 3 : Three associated symptoms; 4: Four associated symptoms.

Table I. Aura types considering group ages (\%); $p>0.05$.

\begin{tabular}{lllll}
\hline & $<6$ yrs & $7-10$ yrs & $11-14$ yrs & $>15$ yrs \\
\hline Visual & 8.5 & 43.5 & 36.2 & 4.9 \\
Sensitivity & 1.2 & 3.06 & 1.2 & 0.0 \\
Motor & 0 & 0 & 0.6 & 0.0 \\
Aphasia & 0 & 0.6 & 0 & 0.0 \\
\hline
\end{tabular}

When the ICHD-II criteria were used, a final diagnosis of MTA was obtained in $15.3 \%$ of patients, pMTA in $13.4 \%$, and TAH in $61.8 \%$, while in in $9.5 \%$ of patients the diagnosis was undetermined (Figure 2). pMTA patients could not be included in 
the MTA group, since all of them showed aura lasting less than $5 \mathrm{~min}$ and $65 \%$ presented pain duration shorter than $1 \mathrm{~h}$. Among the TAH patients, 94.3\% showed a too-short (5-60 min) pain duration.

When the ICHD-3 beta criteria were applied, MTA was diagnosed in $77.7 \%$ of patients and pMTA in $13.4 \%$, with a positive predictive value (PPV) of $85 \%$, while the diagnosis was undetermined in $9.5 \%$ of patients. The pMTA patients did not fulfil the criteria for MTA diagnosis, since all of them (100\%) presented aura duration shorter than 5 minutes.

The patients with undetermined diagnosis were the same independently of the use of either ICHD-II or ICHD-3 beta criteria. They had had less than two headache attacks with aura. We found no patients with aura without headache (Figure 2).

Table 2. Aura duration considering groups ages (\%). min: minutes; $p>0.05$.

\begin{tabular}{llccl}
\hline & $<6$ yrs & $7-10$ yrs & $11-14$ yrs & $>15$ yrs \\
\hline$<5$ min & 2.1 & 7.9 & 8.7 & 0 \\
$5-10$ min & 5.8 & 15.9 & 15.9 & 3.6 \\
$10-30 \mathrm{~min}$ & 2.1 & 15.2 & 9.4 & 1.4 \\
$30-60 \mathrm{~min}$ & 0 & 7.2 & 2.9 & 0.0 \\
$>120 \mathrm{~min}$ & 0 & 0.7 & 0.7 & 0.0 \\
\hline
\end{tabular}

Table 3. Aura timing considering groups ages (\%); $p>0.05$.

\begin{tabular}{llccl}
\hline & $<6$ yrs & $7-10$ yrs & II-14 yrs & $>15$ yrs \\
\hline Pre-ictal & 5.7 & 32.5 & 26.8 & 4.1 \\
Ictal & 1.6 & 11.3 & 6.5 & 1.6 \\
Post-ictal & 0.0 & 5.7 & 4.1 & 0 \\
\hline
\end{tabular}

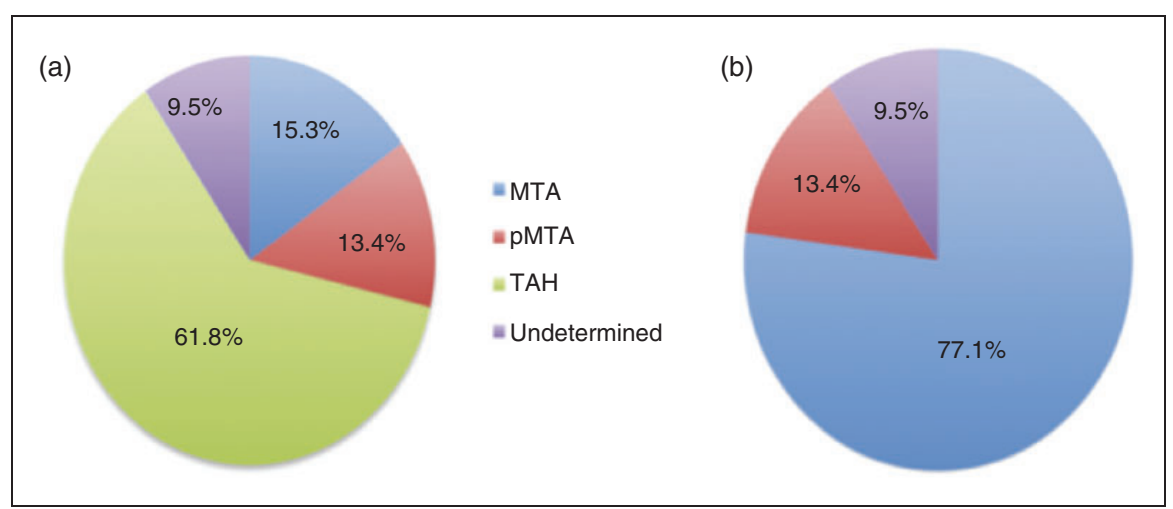

Figure 2. Comparison between diagnoses made using either the ICHD-II (a) or ICHD-III beta (b) criteria.
For the regression analysis, we considered the diagnoses obtained using the ICHD-3 beta criteria. We found that phonophobia and photophobia were associated with MTA and pMTA $(p<0.001)$. Aura characteristics did not correlate to pain characteristics, gender, or age $(p>0.05)$.

\section{Discussion}

Our study addressed analysis of the clinical characteristics of migrainous aura in a pediatric population and tested the diagnostic power of the new ICHD-3 beta criteria for the diagnosis of MWA.

As for the analysis of the aura features, we found that the visual symptoms are more frequent $(93 \%)$ than somatosensory, motor, and speech disturbances. As in adults (15), aura anticipates headache onset in most cases $(69.1 \%)$ and its duration ranges from 5 to $60 \mathrm{~min}$. When we divided our patients into four different age groups ( $<6$ years, $7-10$ years, $11-14$ years, $>15$ years), no difference in the aura characteristics were found between these groups.

Lanzi et al. reported a group of 47 young migraines with aura patients. Visual and sensory disturbances were the most commonly described symptoms; a motor deficit was present in 10 out of their 47 cases. Other disturbances such as vertigo attacks, aphasia, spatial disorientation, loss of consciousness or a decreased level of consciousness with the appearance of automatic movements, were much rarer (16). Balottin et al. conducted a prospective study in 45 patients affected by migraine with aura, evaluating clinical characteristics and aura evolution during attack. The aura propagated slowly, with a pattern corresponding to a cortical pathway in the postero-anterior direction in $71 \%$ of their patients, while it was only visual in $40 \%$. Headache had a better prognosis in terms of frequency of the attacks, when symptoms 
kept being only visual (17). In disagreement with these results, in a study of 137 patients Termine et al. concluded that patients experiencing only visual aura had a lower aura remission rate compared with those with visual, sensory, and aphasic aura symptoms (36.8\% vs. $61.5 \%$ ). Furthermore, a shorter headache duration $(<12$ hours) and the presence of EEG abnormalities at baseline were significant predictors of aura remission at follow-up (17).

Taga et al. recently reported 283 instances of MWA with paediatric onset that were compared with adult onset. In both groups of patients, visual aura was the most common type of aura, followed by sensory and speech disturbances. In the paediatric group, aura without headache was significantly less frequent (1.8 vs. $5.3 \%$ ); furthermore, headache had migraine characteristics in a higher proportion of cases (90.1 vs. $82.6 \%$ ). A family history of MWA was significantly more frequent among cases with paediatric onset $(31.1 \%$ vs. $16.9 \%$ ). Males, but not females with paediatric MWA more frequently had a comorbid migraine without aura (27.6 vs. $16.8 \%)$. Among cases with paediatric onset, we did not find any significant differences between males and females (18).

Genizi et al. reported 262 migrainous children, of whom $26.2 \%$ had MWA. Multiple logistic regression analysis revealed that older age $(p<0.02)$, family history of aura $(p<0.02)$, and (lack of) TTH $(p<0.01)$ were significant predictors of aura, whereas gender was not significant $(p>0.20)$. Children who had migraine with aura were less likely to have mixed headaches than children who did not have aura (OR: 0.26 , $95 \%$ CI: $0.11-0.63 ; p<0.01)(19)$.

Compared to the previous studies, we investigated the largest population of migraine children and adolescents experiencing aura. Our results confirm the aura characteristics described in previous studies and show that they are not different from those found in adults $(1,15)$. As for the headache characteristics associated with aura symptoms, our patients more often experienced a throbbing fronto-temporal pain, lasting less than 4 hours $(94.5 \%)$ and usually associated with photophobia $(64.6 \%)$ and phonophobia (55\%). In our population, the average frequency of migraine attacks was quite low (less than four attacks per month in $76.8 \%$ of patients).

As far as the diagnostic criteria are concerned, we found that the rate of MTA diagnosis is higher with the new than with the previous ICHD criteria. While the ICHD-II distinguishes MTA from THA depending on the pain features, in the ICHD-3 beta the headache features are not relevant for the diagnosis of MTA.
This novelty provides a significant benefit for the diagnosis of MWA in paediatric patients, where the headache attack duration can be shorter than in adults (12). As consequence of this change, all our patients classified as TAH according to the ICHD-II shifted to the MTA group when ICHD-3 beta criteria were applied.

Compared to the ICHD-II, the third version of the ICHD criteria did not reduce the number of patients with a diagnosis of pMTA, since the shortest duration of aura symptoms was kept at 5 minutes. However, this excludes more than $10 \%$ of patients from the diagnosis of MTA.

\section{Limitations of the study}

Our study shows some limitations that are to be considered. First, our results are issued from a retrospective study, thus the new criteria need to be confirmed and validated in a prospective multicentre trial. However, it is to be underlined that we had the possibility to describe the largest series of children and adolescents with MWA. Second, we are aware that determining the duration of the pain attack and/or aura symptoms in children, especially the younger ones, may be subject to mistakes. This is because it can be difficult to understand when the attack and/or the aura truly end. Furthermore, considering the child's behaviour (recommencement of normal activity) can lead to bias in judging that pain and/or aura symptoms ended while the child still had mild pain. This possibility may have artificially shortened both the headache attack and aura duration in some of our youngest children. Third, a further limitation concerns the difficulty in recognising aura symptoms in very young children who cannot communicate or describe them. Therefore, although the diagnostic workup for children suffering from headache is similar to that used for adults, certain peculiarities must be considered (e.g. non-verbal behaviours).

\section{Conclusions}

In conclusion, our paediatric population shows aura features that do not depend on age and are similar to those of adult patients. However, a final diagnosis of MTA could be less often reached when using the ICHD-II criteria, which consider headache duration. In this view, the ICHD-3 beta criteria offer the advantage of not considering headache features, including pain duration, thus increasing the MTA diagnosis rate. 


\section{Clinical implications}

- The paediatric population shows aura features that do not depend on age and are similar to those of adult patients.

- Children with migraine showed a shorter duration of attacks than adults.

- ICHD-3 beta criteria offer the advantage of not considering headache features, including pain duration, thus increasing the MTA diagnosis rate.

\section{Declaration of conflicting interests}

The authors declared no potential conflicts of interest with respect to the research, authorship, and/or publication of this article.

\section{Funding}

The authors received no financial support for the research, authorship, and/or publication of this article.

\section{References}

1. Headache Classification Committee of the International Headache Society (IHS). The International Classification of Headache Disorders, 3rd edition (beta version). Cephalalgia 2013; 33: 629-808.

2. Stovner LJ, Hagen K, Jensen R, et al. The global burden of headache: A documentation of headache prevalence and disability worldwide. Cephalalgia 2007; 27: 193-210.

3. Jensen R and Stovner LJ. Epidemiology and comorbidity of headache. Lancet Neurol 2008; 7: 354-361.

4. Metsähonkala L and Sillanpää M. Migraine in children an evaluation of the IHS criteria. Cephalalgia 1994; 14 : 285-290.

5. Laurell K, Larsson B and Eeg-Olofsson O. Prevalence of headache in Swedish schoolchildren, with a focus on tension-type headache. Cephalalgia 2004; 24: 380-388.

6. Abu-Arafeh I1, Razak S, Sivaraman B, et al. Prevalence of headache and migraine in children and adolescents: A systematic review of population-based studies. Dev Med Child Neurol 2010; 52: 1088-1097.

7. Merikangas KR. Contributions of epidemiology to our understanding of migraine. Headache 2013; 53: 230-246.

8. Gergont A, Gajda B and Wesołowska E. Results of autonomic testing in children with migraine with aura, including visual and sensory. Przegl Lek 2016; 73: $143-147$.
9. Rastogi RG, VanderPluym $\mathbf{J}$ and Lewis KS. Migrainous aura, visual snow, and "Alice in Wonderland" Syndrome in childhood. Semin Pediatr Neurol 2016; 23: 14-17.

10. Kienbacher C, Wöber C, Zesch HE, et al. Clinical features, classification and prognosis of migraine and tension-type headache in children and adolescents: A long-term follow-up study. Cephalalgia 2006; 26: 820-830.

11. Lima MM, Padula NA, Santos LC, et al. Critical analysis of the International Classification of Headache Disorders diagnostic criteria (ICHD I-1988) and (ICHD II-2004), for migraine in children and adolescents. Cephalalgia 2005; 25: 1042-1047.

12. Torriero R, Capuano A, Mariani R, et al. Diagnosis of primary headache in children younger than 6 years: A clinical challenge. Cephalalgia 2017; 37: 947-954.

13. Winner P. How do we diagnose migraine and childhood periodic syndromes? Curr Pain Headache Rep 2005; 9: 345-350.

14. Tarantino S, Capuano A, Torriero R, et al. Migraine equivalents as part of migraine syndrome in childhood. Pediatr Neurol 2014; 51: 645-649.

15. Russell MB and Olesen J. The genetics of migraine without aura and migraine with aura. Cephalalgia 1993; 13 : 245-248.

16. Lanzi G, Balottin U and Borgatti R. A prospective study of juvenile migraine with aura. Headache 1994; 34: 275-278.

17. Balottin U, Borgatti R, Zambrino CA, et al. Clinical characteristics and long-term outcome of migraine with aura in children and adolescents. Dev Med Child Neurol 1997; 39: 26-30.

18. Taga A, Russo M, Genovese A, et al. Pediatric migraine with aura in an Italian case series. Neurol Sci 2017; 38: 185-187.

19. Genizi J, Khourieh Matar A, Schertz M, et al. Pediatric mixed headache - the relationship between migraine, tension-type headache and learning disabilities - in a clinicbased sample. J Headache Pain 2016; 17: 42-46. 